\title{
Randomized Trial of Repetitive Transcranial Magnetic Stimulation for Apathy and Depression in Parkinson's Disease
}

\author{
Hiroaki Oguro $^{{ }^{*}}$, Tomonori Nakagawa ${ }^{1}$, Shingo Mitaki ${ }^{1}$, Masaki Ishihara ${ }^{2}$, Keiichi Onoda ${ }^{1}$ and Shuhei Yamaguchi ${ }^{1}$ \\ ${ }^{1}$ Department of Neurology, Faculty of Medicine, Shimane University, Shimane, Japan \\ ${ }^{2}$ Department of Neurology, Tokyo Metropolitan Health and Medical Treatment Corporation, Toshima Hospital, Tokyo, Japan \\ *Corresponding author: Hiroaki Oguro, 89-1 Enya-cho, Izumo, Shimane 693-8501, Japan, Tel: +81-853-20-2199; E-mail: oguro@med.shimane-u.ac.jp
}

Received date: Aug 20, 2014, Accepted date: Oct 27, 2014, Published date: Oct 31, 2014

Copyright: (c) 2014 Oguro $\mathrm{H}$, et al. This is an open-access article distributed under the terms of the Creative Commons Attribution License, which permits unrestricted use, distribution, and reproduction in any medium, provided the original author and source are credited.

\begin{abstract}
Objective: Repetitive transcranial magnetic stimulation (rTMS) has been reported to improve motor function and depression in Parkinson's disease (PD) patients, but there has been only one randomized controlled trial for apathy. We evaluated the efficacy of rTMS for apathy and depression in Parkinson's disease.

Methods: Fifteen PD patients received real rTMS $(5 \mathrm{~Hz}, 500$ pulses/day) and placebo stimulation over the supplementary motor area (SMA) for each 5 days with total amount of 2500 real pulses and 2500 placebo pulses, using a randomized real-first or placebo-first protocol. The modified apathy scale, the Zung Self-rating Depression Scale (SDS) and Unified Parkinson's Disease Rating Scale (UPDRS) were used to assess apathy, depression and clinical status before and after each stimulative treatment.

Results: Real rTMS improved the apathy score by 3 points $(P<0.05)$ compared to the baseline, while placebo stimulation produced no improvement, irrespective of the order of treatment. Real rTMS also improved the depression scale, SDS by 5 points $(P<0.05)$ compared to the baseline, while placebo stimulation was ineffective. Combined analysis confirmed that real rTMS was significantly superior to placebo stimulation in apathy and depression $(p<0.05)$. Real rTMS also improved UPDRS by 10 points $(P=0.001)$, while placebo stimulation was ineffective. No side effects were observed in either real rTMS or placebo stimulation. Clinical factors including age, gender, disease duration, UPDRS score pre-rTMS, and daily dose of L-DOPA did not influence the improvement of UPDRS and apathy scores by real rTMS.
\end{abstract}

Conclusions: rTMS over the SMA appears to be effective for treatment of apathy and depression in PD patients in addition to UPDRS.

Keywords: Apathy; rTMS; Parkinson's disease; Depression, SDS, UPDRS

\section{Introduction}

Alterations in mood, depression and apathy are the most frequent non-motor symptoms in Parkinson's disease (PD) [1]. Repetitive transcranial magnetic stimulation (rTMS) has been used as a noninvasive stimulation for Parkinson's disease, and has been modestly reported to improve motor function and bradykinesia compared with sham stimulation [2,3]. In addition, high-frequency rTMS over the left dorsolateral prefrontal cortex (DLPFC) was found to improve depression including PD patients with mood disorders, and low-frequency rTMS on the right DLPFC was also effective [4-9]. Apathy is considered a prevalent symptom with lack of motivation, and 17 to $70 \%$ of patients with PD also suffer from apathy [10]. However, there has been a few randomized controlled trial with welldefined inclusion criteria for the treatment of depression and apathy in PD patients $[8,11,12]$. Therefore, we examined whether rTMS improves apathy and depression in PD patients, using a randomized cross-over design of real and placebo stimulus sessions.

\section{Patients and Methods}

Participants included 15 subjects with Parkinson's disease (PD) (mean age; 72.7, $\mathrm{SD}=8.4$ ). The study protocol was approved by Shimane University Institutional Committee on Ethics (registered in the study number 231). Written informed consent was obtained from every participant before intervention. Their disease duration ranged from 33 to 218 months (mean 89.3 months, $S D=48.9$ ). All subjects underwent neurological examination and their total score on the unified Parkinson's disease rating scale (UPDRS) was estimated. Apathy was evaluated according to the modified apathy scale (our revised version in Japanese [13]). Depression was also evaluated with the Zung Self-rating Depression Scale (SDS) [14]. Assessment of apathy, depression and UPDRS was performed before and after both real and placebo stimulations. Apathy scale, SDS and UPDRS were blind to stimulative allocation, real or placebo stimulation, and were obtained by blind raters. The patients continued to take their usual medications during the study. Medication for PD was only restricted to L-DOPA, because dopamine receptor agonist might influence on apathy and alter dopamine reward system [15-17].

The rTMS was performed under two conditions (real rTMS and placebo stimulation). For real rTMS, a figure-of-eight-shaped coil connected to a magnetic stimulator (Magstim Rapid; The Magstim 
Citation: Oguro H, Nakagawa T, Mitaki S, Ishihara M, Onoda K, et al (2014) Randomized Trial of Repetitive Transcranial Magnetic Stimulation for Apathy and Depression in Parkinson's Disease. J Neurol Neurophysiol 5: 242. doi:10.4172/2155-9562.1000242

Page 2 of 6

Co.UK) was attached on the cranial surface over the SMA vertically in relation to the parasagittal plane. We selected the supplementary motor area (SMA) as the stimulus site, because SMA stimulation has been reported to improve motor function in PD patients. The stimulus site was set $3 \mathrm{~cm}$ anterior to the site of the anterior tibial muscle. Stimulus intensity, expressed as a percentage of the maximum stimulator output, was set at $110 \%$ resting motor threshold (MT) for the right abductor of pollicis brevis muscle [18]. One TMS session consisted of 10 trains of $10 \mathrm{~s}$ duration with $5 \mathrm{~Hz}$ frequency; 5 trains were applied over each hemisphere. Placebo stimulation was delivered with the coil angled at $90^{\circ}$, so that only the edge of the coil rested on the scalp. The stimulus parameters were the same as in real rTMS. The patients were randomized into real-first $(\mathrm{n}=8)$ and placebo-first $(\mathrm{n}=$ 7) groups. Each of real rTMS and placebo stimulation was delivered for five days, and wash out period was set for two days between real and placebo stimulation (Figure 1).

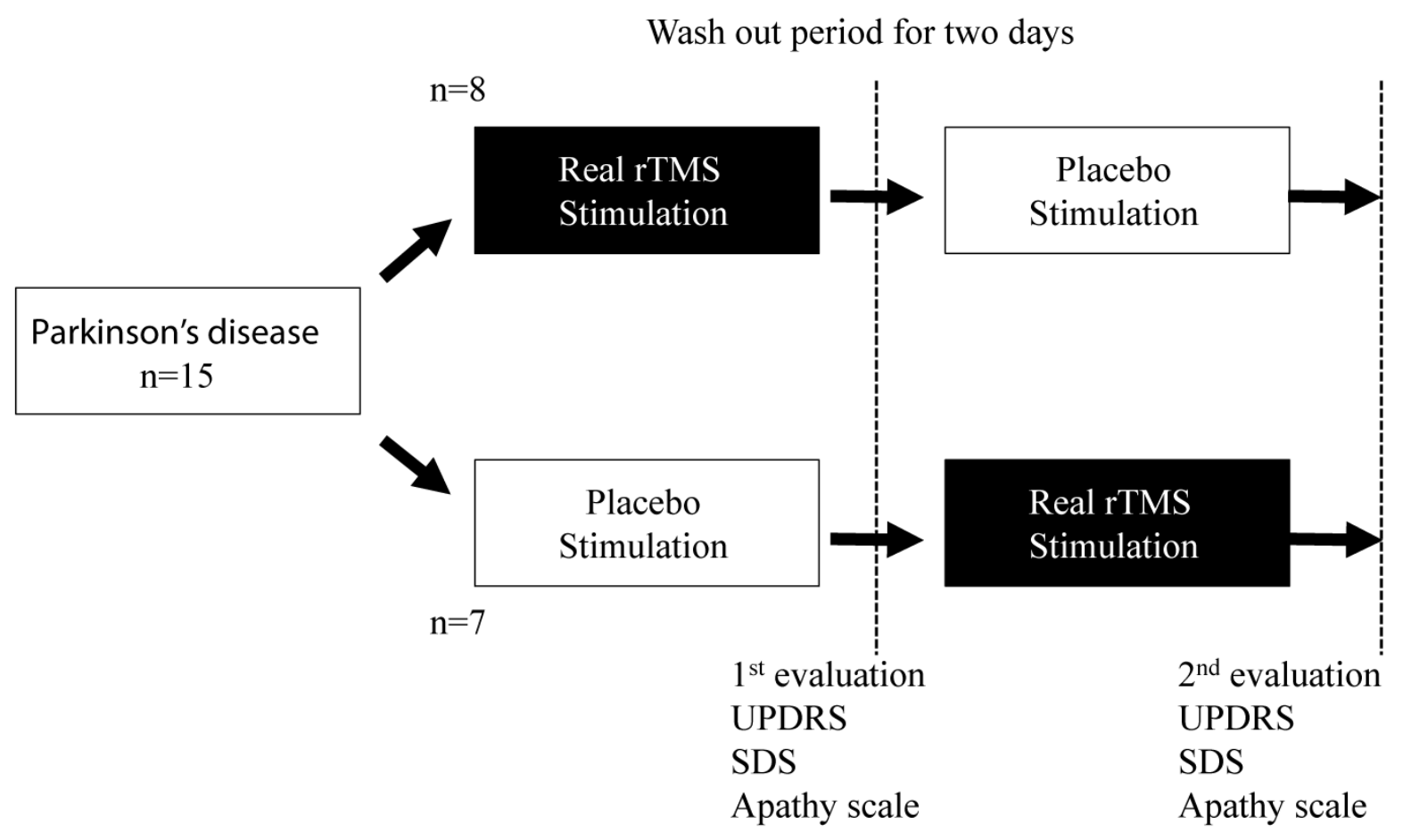

Figure 1: The rTMS was performed under two conditions (real rTMS and placebo stimulation) by randomized and cross over designed study. The patients were randomized into real-first $(n=8)$ and placebo-first $(n=7)$ groups. SDS: Zung Self-rating Depression Scale, UPDRS: the unified Parkinson's disease rating scale

The Wilcoxon signed ranks test was used for comparison of apathy scales, SDS and UPDRS before and after stimulation for real rTMS and for placebo stimulus session. Group comparison was performed using repeated-measures ANOVA with UPDRS, apathy scale and SDS as a within-subjects factor and real or placebo stimulation as a betweensubjects factor. The Spearman correlation coefficient was used to calculate the relationship among the changes in apathy scale, SDS and UPDRS scores in each session (real rTMS and placebo stimulation). To examine which clinical factors contribute to the effects of rTMS therapy, we assessed the contributions of age, gender, disease duration (months), UPDRS score pre-rTMS, and daily dose of L-DOPA by means of logistic regression analysis. A value of $\mathrm{P}<0.05$ was considered statistically significant.

\section{Results}

Baseline characteristics of 2 groups (procedure of stimulation), real placebo group and placebo real group. Mean age, gender, length of illness, daily dosement of L-DOPA, total UPDRS on pre-stimulation, MMSE, apathy, depression and motor threshold intensity were all not different between two groups. Stimulation intensity at $110 \%$ resting motor threshold ranged from 48 to 93 (mean value is 73 intensity, $\mathrm{SD}=14$ ) (Table 1). Total UPDRS score ranged from 20 to 88 (mean 46, $\mathrm{SD}=17$ ), and all participants were usually on state with no antipsychotic drugs.

\begin{tabular}{|l|l|l|l|}
\hline & $\mathbf{1}^{\text {st }}$ Real $\& 2^{\text {nd }}$ placebo group $(\mathbf{n}=\mathbf{8})$ & $\mathbf{1}^{\text {st }}$ Placebo \& $\mathbf{2}^{\text {nd }}$ Real group $(\mathbf{n}=\mathbf{7})$ & $\mathbf{P}$ \\
\hline Mean age (years) & $70.0 \pm 8.4$ & $76.0 \pm 7.7$ & $0.163^{*}$ \\
\hline Male (\%) & $2(25.0)$ & $2(28.5 \%)$ & $0.9999 \S$ \\
\hline
\end{tabular}


Citation: Oguro H, Nakagawa T, Mitaki S, Ishihara M, Onoda K, et al (2014) Randomized Trial of Repetitive Transcranial Magnetic Stimulation for Apathy and Depression in Parkinson's Disease. J Neurol Neurophysiol 5: 242. doi:10.4172/2155-9562.1000242

Page 3 of 6

\begin{tabular}{|l|l|l|l|}
\hline Length of illness (months) & $78 \pm 35$ & $102 \pm 62$ & $0.6425^{\star}$ \\
\hline L-DOPA (mg/day) & $444 \pm 129$ & $364 \pm 165$ & $0.3794^{*}$ \\
\hline UPDRS total & $52 \pm 17$ & $39 \pm 16$ & $0.0638^{*}$ \\
\hline MMSE & $27 \pm 4$ & $27 \pm 3$ & $0.8582^{\star}$ \\
\hline apathy scale & $14 \pm 7$ & $19 \pm 8$ & $0.3846^{*}$ \\
\hline apathy patient & $2(25 \%)$ & $4(57 \%)$ & $0.4385 \S$ \\
\hline SDS (depression) & $48 \pm 7$ & $44 \pm 10$ & $0.324^{*}$ \\
\hline $110 \%$ MT intensity & $69 \pm 17$ & $77 \pm 8$ & $0.3532^{*}$ \\
\hline
\end{tabular}

Table 1: Baseline characteristics of 2 groups (procedure of stimulation), real placebo group and placebo real group with mean age, gender, length of illness, daily dosement of L-DOPA, total UPDRS on pre-stimulation, MMSE, apathy, depression and motor threshold intensity. Values are mean \pm SD, UPDRSthe unified Parkinson's disease rating scale, SDS :Zung Self-rating Depression Scale, MMSEmini-mental state examination, MT: Motor threshold *: Mann-Whitney U test, §: Fisher's exact test.

Apathy scale before rTMS ranged from 3 to 29 (mean 16, SD=8), and 6 participants $(40 \%)$ with being more than 16 were diagnosed by apathy. Real rTMS improved the apathy scale by 3 points compared to the baseline ( $p r e=18$, post $=15, \mathrm{p}<0.05$ ), whereas placebo stimulation worsened the score by 3 points (pre $=15$, post $=18, \mathrm{p}<0.05$ ). When the changes of apathy scale between the two sessions (real rTMS and placebo stimulation) were evaluated with repeated-measures ANOVA, real rTMS produced a significant improvement in apathy score compared with placebo stimulation $(\mathrm{p}<0.05)$ (Figure 2). Stimulative procedure of real and placebo have no significant interaction (stimulation $\mathrm{x}$ timing: $\mathrm{F}=1.826, \mathrm{p}=0.1818$ ). SDS before $\mathrm{rTMS}$ ranged from 25 to 60 (mean 46, SD=9), and 6 participants (40\%) with being more than 50 were diagnosed by depression. SDS statistically changed after real TMS (pre $=47$, post $=42, \mathrm{p}<0.05$ ) and placebo stimulation (pre $=44$, post $=46, \mathrm{p}<0.05$ ). When the changes of SDS between the two sessions (real rTMS and placebo stimulation) were evaluated with repeated-measures ANOVA, real rTMS produced a significant improvement in SDS compared with placebo stimulation $(\mathrm{p}<0.05)$ (Figure 3). Stimulative procedure of real and placebo have no significant interaction (stimulation $\mathrm{x}$ timing: $\mathrm{F}=2.963, \mathrm{p}=0.079$ ).

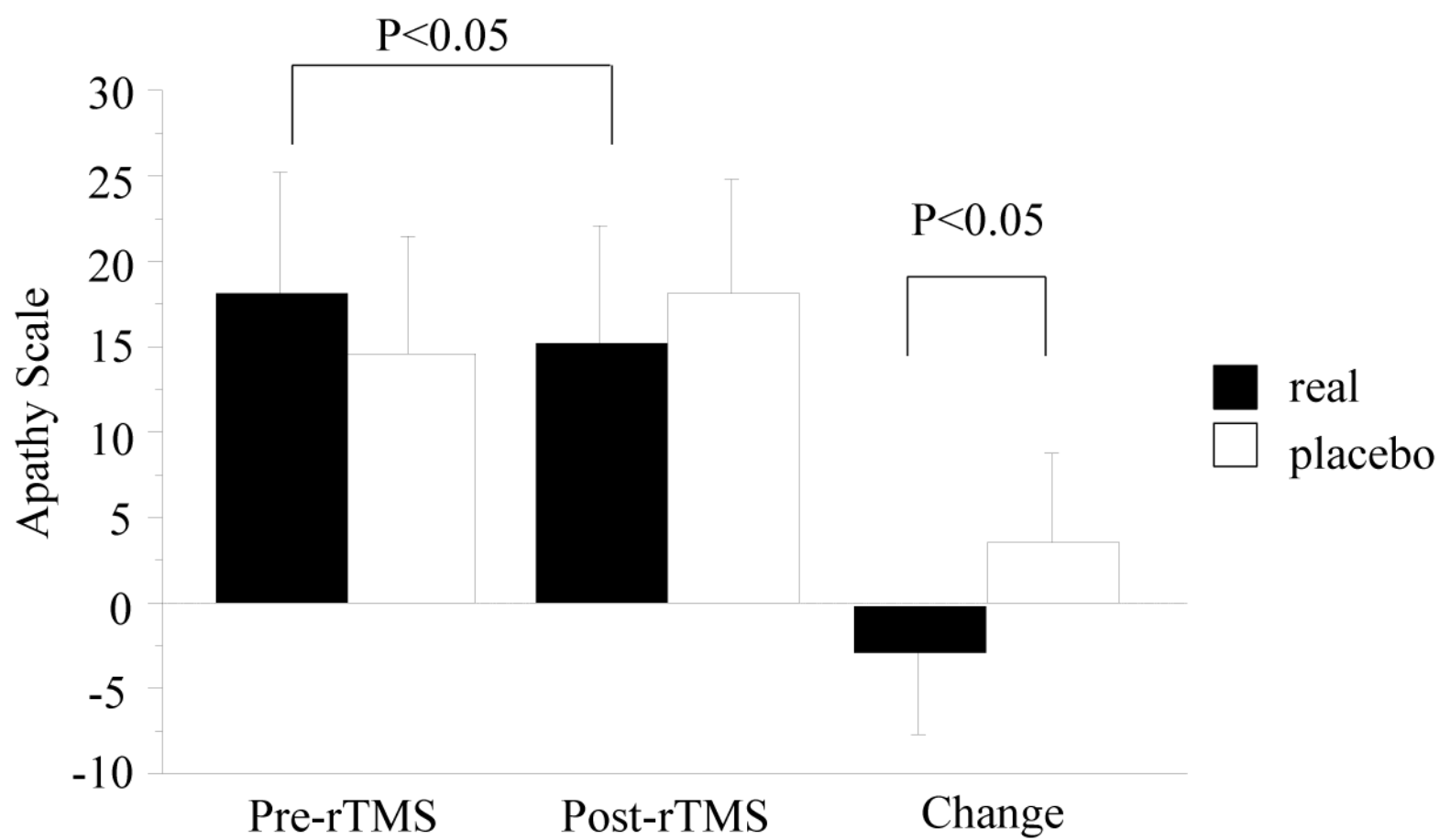

Figure 2: The effects of real rTMS and placebo stimulation on apathy scale score in 15 Parkinson's disease patients. 
Citation: Oguro H, Nakagawa T, Mitaki S, Ishihara M, Onoda K, et al (2014) Randomized Trial of Repetitive Transcranial Magnetic Stimulation for Apathy and Depression in Parkinson's Disease. J Neurol Neurophysiol 5: 242. doi:10.4172/2155-9562.1000242

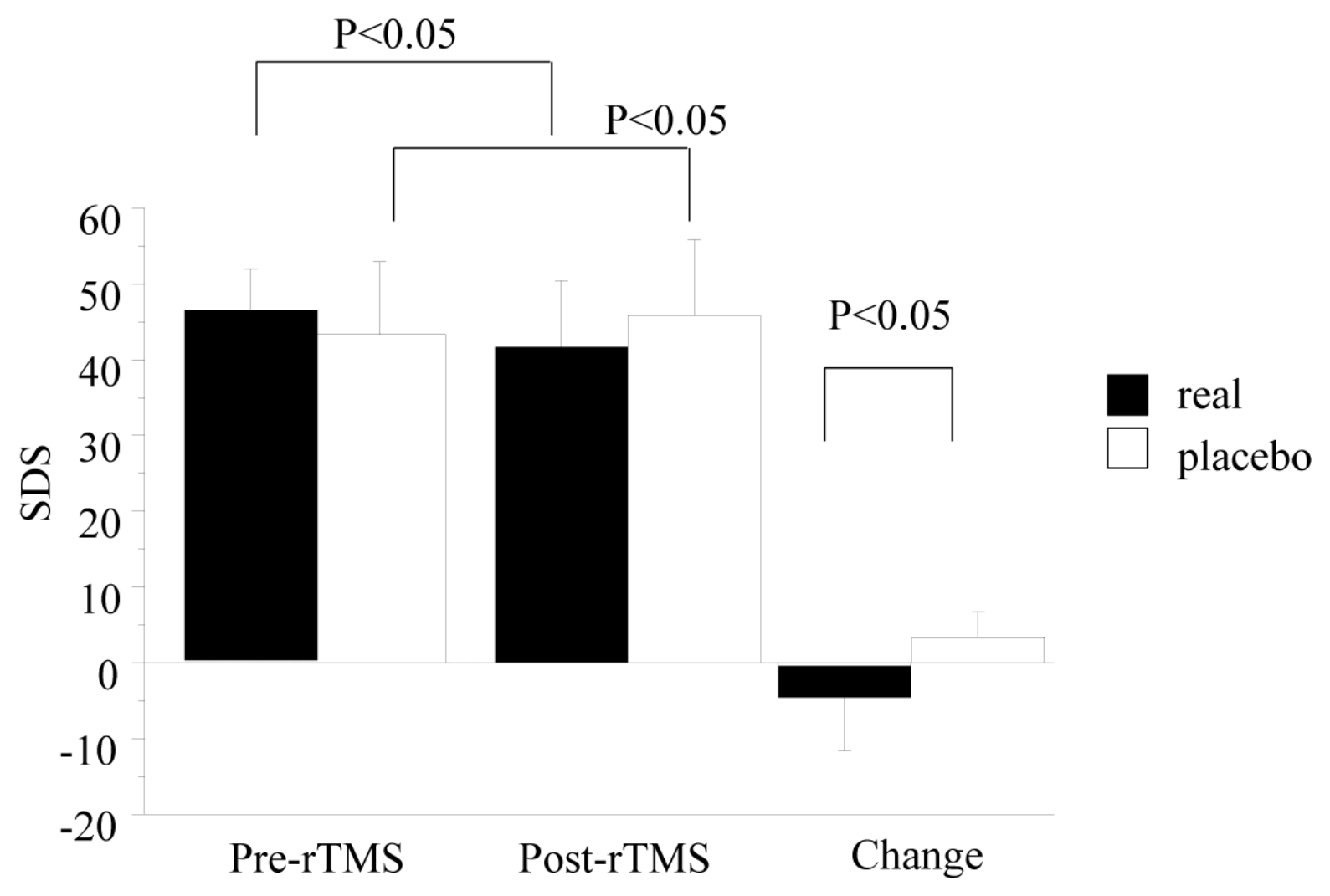

Figure 3: The effects of real rTMS and placebo stimulation on SDS in 15 Parkinson's disease patients. SDS: Zung Self-rating Depression Scale

Real rTMS improved UPDRS by 10 points (pre $=46$, post $=36$, $\mathrm{P}=0.001$ ), but UPDRS did not change after placebo stimulation (pre $=$ 43 , post $=43$ ). When the changes of UPDRS between the two sessions were compared with repeated-measures ANOVA, real rTMS produced a significant improvement of UPDRS compared with placebo stimulation $(\mathrm{p}<0.01)$ (Figure 4). Stimulative procedure of 1 st real and 2nd placebo was significantly more effective than 1 st placebo and 2 nd real with interaction (stimulation $\mathrm{x}$ timing: $\mathrm{F}=7.306, \mathrm{p}=0.001$ ). No side effects were observed in either real or placebo stimulation. Clinical factors (i.e., age, gender, disease duration (months), UPDRS score prerTMS, and daily dose of L-DOPA) did not influence the improvement of UPDRS and apathy scores by real rTMS. Between the two sessions there was no correlation among the changes in apathy scale, SDS and UPDRS scores.

\section{Discussion}

Apathy is defined as lack of motivation, manifested as diminished goal-directed behavior and cognition and decreased emotional engagement. It reduces the quality of life of PD patients with increasing functional dependency, as well as caregivers $[19,20]$. Dysfunction of the frontal-subcortical circuits is thought to be a cardinal pathophysiologal feature of apathy in PD patients, because a reduction in dopaminergic afferents to the striatum disturbs connections between frontal lobe, caudate, anterior cingulate circuits and the basal ganglia [21,22]. This is also consistent with our report that a dopamine $\mathrm{D} 2 / 3$ receptor agonist was effective for the treatment of apathy [23]. Further, we have shown that cerebral blood flow is reduced in the dorsolateral frontal lobe and basal ganglia of apathetic stroke patients $[13,24]$. Hypoactivity in these regions appears to contribute to the emergence of apathy symptoms. Mottaghy et al. reported that rTMS at the DLPFC caused a local increase of regional cerebral blood flow in several neocortical areas [25]. Boggio et al. reported that rTMS stimulation improves the score in the Stroop test, which reflects attention and executive function associated with the frontal lobe [9]. Further, the score in the Stroop test was significantly correlated with the apathy scale score in PD and depressed patients $[26,27]$. These reports also suggest that rTMS may be effective to improve frontal lobe functions.

We adopted the method described in the University Tokyo research group's reports, in which the SMA was stimulated for the treatment of $\mathrm{PD}[2,3,12]$. The SMA is functionally impaired in PD patients, because of decreased positive efferent feedback involving the motor circuit. High-frequency rTMS activates neurons of the SMA and ameliorates motor circuit dysfunction [2,3]. The majority of previous studies have employed a rTMS intensity of $110 \%$ of MT for the treatment of depression, as we did here [6]. Our findings are in agreement with the University Tokyo research group's reports as regards UPDRS improvement in response to rTMS over the SMA [2,12]. Although four reports showed rTMS on let DLPFC in PD are effective on depressed PD [6-9], our rTMS on SMA had been also effective on depression. Our stimulus intensity $5 \mathrm{~Hz}$ is lower than these reports 
Citation: Oguro H, Nakagawa T, Mitaki S, Ishihara M, Onoda K, et al (2014) Randomized Trial of Repetitive Transcranial Magnetic Stimulation for Apathy and Depression in Parkinson's Disease. J Neurol Neurophysiol 5: 242. doi:10.4172/2155-9562.1000242

Page 5 of 6

$(10 \sim 15 \mathrm{~Hz})$ and our stimulus position SMA is different from left DLPFC in four reports. Although we could not clearly explain why placebo stimulation worsened depression scale, only placebo stimulation without any drug control might affect depressive state.

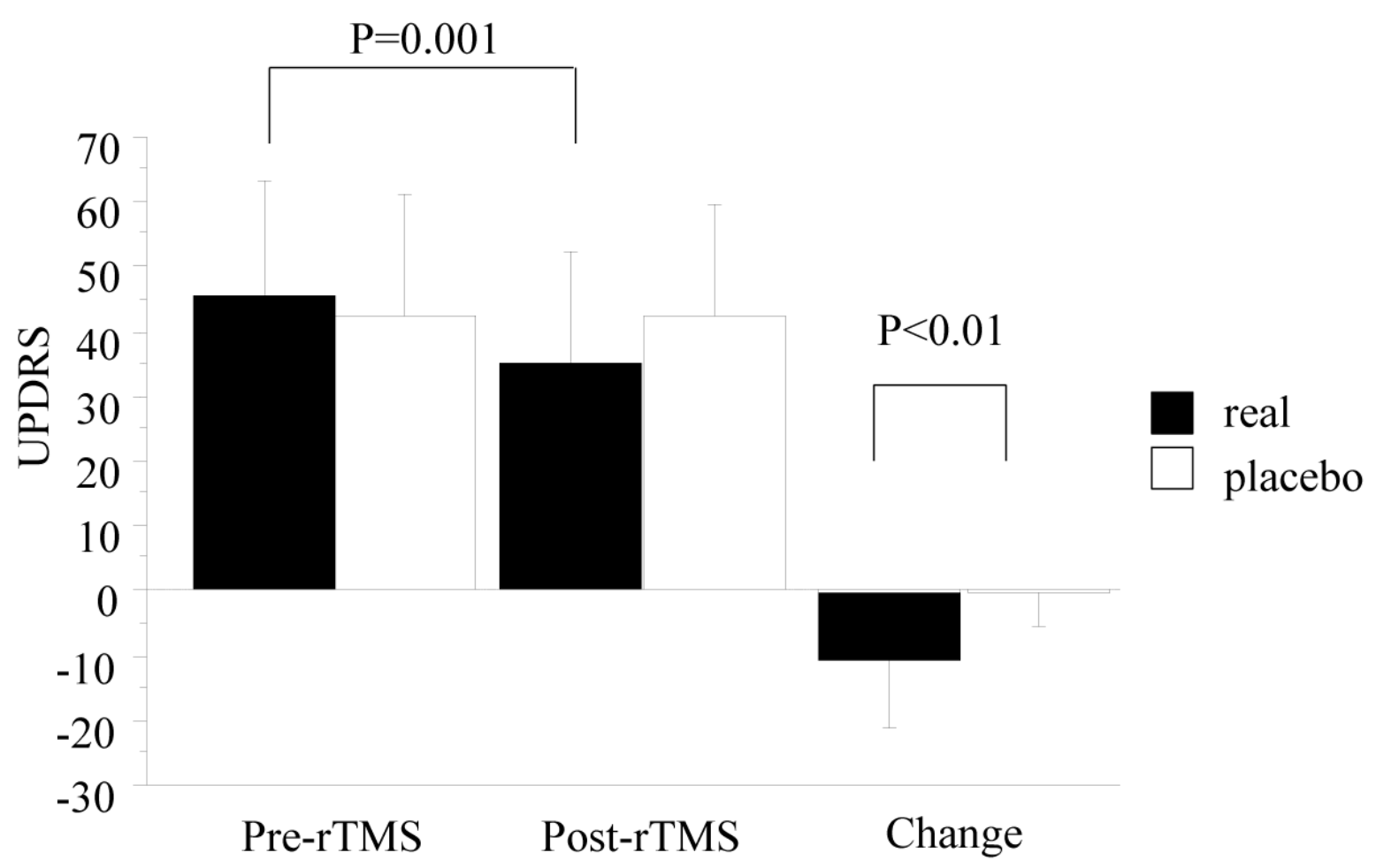

Figure 4: The effects of real rTMS and placebo stimulation on UPDRS in 15 Parkinson's disease patients. UPDRS: the unified Parkinson's disease rating scale

There is some uncertainty as to whether stimulation of the SMA can cause activation of the frontal-subcortical network, which is compromised in patients with apathy. SMA has been reported to be activated in association with DLPFC activation in several cognitive activities, such as the Stroop task [28] and retrieval of motor memory following interleaved practice and skill learning [29], suggesting that there is functional connectivity between the SMA and DLFPC. We speculate that SMA stimulation by rTMS indirectly up-regulates neuronal activity in the frontal-subcortical circuit and contributes to improvement of apathy and depression in PD patients. The degree of improvement in apathy and depression was not correlated with UPDRS score in our study, so it is unlikely that alleviation of apathy and depression was caused by improved motor performance [7].

One randomized controlled trial for the treatment of $1 \mathrm{~Hz}$ or $10 \mathrm{~Hz}$ rTMS of apathy in $70 \mathrm{PD}$ patients showed no efficacy [12]. This research design was a weekly intervention with 8 times stimulation with daily 1000 pulses (total amount of 8000 pulses for 8 weeks). It was different form our rTMS stimulative frequency $5 \mathrm{~Hz}$ with daily 500 pulses and total amount of 2500 pulses for 5 days. So our real rTMS design with every 5 days (2500 pulses per a week) might be slightly stronger than theirs (1000 pulses per a week). In rTMS intervention for PD with apathy, our report is second randomized trial with original stimulative method on every day compared to Shirota's first report with weekly rTMS intervention [12].
Our rTMS session is consists of twelve days in admission and we set wash out period for two days between real and placebo stimulation. The lasting time of rTMS efficacy on motor cortical excitability varied from one hour to one month with various stimulative intensities. It can last one hour by frequency $1 \mathrm{~Hz}, 10$ days by $5 \mathrm{~Hz}$, and one month by $25 \mathrm{~Hz}[30,31]$. However effective time could be modified with various protocol (rTMS frequency, intensity, position and total pulses), definite protocol would be required to determine.

The main limitation of this study is the small sample size. However, the finding that the effect of rTMS was statistically significant even in as few as 15 patients indicates that the effect is robust. Another possible limitation is that rTMS was conducted at a location known to produce an improvement of motor dysfunction as well as apathy. We think a further study that includes a specific protocol to identify optimal brain regions for treatment to improve apathy would be worthwhile. And we need to investigate hippocampus as a treated target which is a central area in modulation of mood and apathy. rTMS with double cone coil might be useful for stimulating deep brain region.

\section{References}

1. Barone P, Antonini A, Colosimo C, Marconi R, Morgante L, et al. (2009) The PRIAMO study: A multicenter assessment of nonmotor symptoms and their impact on quality of life in Parkinson's disease. Mov Disord 24: 1641-1649. 
Citation: Oguro H, Nakagawa T, Mitaki S, Ishihara M, Onoda K, et al (2014) Randomized Trial of Repetitive Transcranial Magnetic Stimulation for Apathy and Depression in Parkinson's Disease. J Neurol Neurophysiol 5: 242. doi:10.4172/2155-9562.1000242

Page 6 of 6

2. Hamada M, Ugawa Y, Tsuji S; Effectiveness of rTMS on Parkinson's Disease Study Group, Japan (2008) High-frequency rTMS over the supplementary motor area for treatment of Parkinson's disease. Mov Disord 23: 1524-1531.

3. Hamada M, Ugawa Y, Tsuji S; Effectiveness of rTMS on Parkinson's Disease Study Group, Japan (2009) High-frequency rTMS over the supplementary motor area improves bradykinesia in Parkinson's disease: subanalysis of double-blind sham-controlled study. J Neurol Sci 287: 143-146.

4. Pascual-Leone A, Rubio B, Pallardó F, Catalá MD (1996) Rapid-rate transcranial magnetic stimulation of left dorsolateral prefrontal cortex in drug-resistant depression. Lancet 348: 233-237.

5. Klein E, Kreinin I, Chistyakov A, Koren D, Mecz L, et al. (1999) Therapeutic efficacy of right prefrontal slow repetitive transcranial magnetic stimulation in major depression: a double-blind controlled study. Arch Gen Psychiatry 56: 315-320.

6. Fregni F, Santos CM, Myczkowski ML, Rigolino R, Gallucci-Neto J, et al. (2004) Repetitive transcranial magnetic stimulation is as effective as fluoxetine in the treatment of depression in patients with Parkinson's disease. J Neurol Neurosurg Psychiatry 75: 1171-1174.

7. Epstein CM, Evatt ML, Funk A, Girard-Siqueira L, Lupei N, et al. (2007) An open study of repetitive transcranial magnetic stimulation in treatment-resistant depression with Parkinson's disease. Clin Neurophysiol 118: 2189-2194.

8. Pal E, Nagy F, Aschermann Z, Balazs E, Kovacs N (2010) The impact of left prefrontal repetitive transcranial magnetic stimulation on depression in Parkinson's disease: a randomized, double-blind, placebo-controlled study. Mov Disord 25: 2311-2317.

9. Boggio PS, Fregni F, Bermpohl F, Mansur CG, Rosa M, et al. (2005) Effect of repetitive TMS and fluoxetine on cognitive function in patients with Parkinson's disease and concurrent depression. Mov Disord 20: 1178-1184.

10. Leentjens AF, Dujardin K, Marsh L, Martinez-Martin P, Richard IH, et al. (2008) Apathy and anhedonia rating scales in Parkinson's disease: critique and recommendations. Mov Disord 23: 2004-2014.

11. Seppi K, Weintraub D, Coelho M, Perez-Lloret S, Fox SH, et al. (2011) The Movement Disorder Society Evidence-Based Medicine Review Update: Treatments for the non-motor symptoms of Parkinson's disease. Mov Disord 26 Suppl 3: S42-80.

12. Shirota Y, Ohtsu H, Hamada M, Enomoto H, Ugawa Y; Research Committee on rTMS Treatment of Parkinson's Disease (2013) Supplementary motor area stimulation for Parkinson disease: a randomized controlled study. Neurology 80: 1400-1405.

13. Okada K, Kobayashi S, Yamagata S, Takahashi K, Yamaguchi S (1997) Poststroke apathy and regional cerebral blood flow. Stroke 28: 2437-2441.

14. ZUNG WW (1965) A SELF-RATING DEPRESSION SCALE. Arch Gen Psychiatry 12: 63-70.

15. Moller JC, Oertel WH, Köster J, Pezzoli G, Provinciali L (2005) Longterm efficacy and safety of pramipexole in advanced Parkinson's disease: results from a European multicenter trial. Mov Disord 20: 602-610.
16. Kéri S, Nagy H, Levy-Gigi E, Kelemen O (2013) How attentional boost interacts with reward: the effect of dopaminergic medications in Parkinson's disease. Eur J Neurosci 38: 3650-3658.

17. Oguro H, Kadota K, Ishihara M, Okada K, Yamaguchi S (2014) Efficacy of pramipexole for treatment of apathy in Parkinson's disease. International Journal of Clinical Medicine 5: 885-889

18. Pascual-Leone A (1994) Akinesia in Parkinson's disease. Shortening of choice reaction time and movement time with subthreshold repetitive transcranial motor cortex stimulation. Neurology 44: 892-898.

19. Marin RS (1991) Apathy: a neuropsychiatric syndrome. J Neuropsychiatry Clin Neurosci 3: 243-254.

20. Pedersen KF, Larsen JP, Alves G, Aarsland D (2009) Prevalence and clinical correlates of apathy in Parkinson's disease: a community-based study. Parkinsonism Relat Disord 15: 295-299.

21. Cummings JL (1993) Frontal-subcortical circuits and human behavior. Arch Neurol 50: 873-880.

22. Dujardin K, Sockeel P, Devos D, Delliaux M, Krystkowiak P, et al. (2007) Characteristics of apathy in Parkinson's disease. Mov Disord 22: 778-784.

23. Kohno N, Abe S, Toyoda G, Oguro H, Bokura H, et al. (2010) Successful treatment of post-stroke apathy by the dopamine receptor agonist ropinirole. J Clin Neurosci 17: 804-806.

24. Yamagata S1, Yamaguchi S, Kobayashi S (2004) Impaired novelty processing in apathy after subcortical stroke. Stroke 35: 1935-1940.

25. Mottaghy FM, Keller CE, Gangitano M, Ly J, Thall M, et al. (2002) Correlation of cerebral blood flow and treatment effects of repetitive transcranial magnetic stimulation in depressed patients. Psychiatry Res 115: 1-14.

26. Feil D, Razani J, Boone K, Lesser I (2003) Apathy and cognitive performance in older adults with depression. Int J Geriatr Psychiatry 18: 479-485.

27. Santangelo G, Vitale C, Trojano L, Longo K, Cozzolino A, et al. (2009) Relationship between depression and cognitive dysfunctions in Parkinson's disease without dementia. J Neurol 256: 632-638.

28. Marek T, Fafrowicz M, Golonka K, Mojsa-Kaja J, Oginska H,et al. (2010) Diurnal patterns of activity of the orienting and executive attention neuronal networks in subjects performing a Stroop-like task: a functional magnetic resonance imaging study. Chronobiol Int 27: 945-958.

29. Lin $\mathrm{CH}$, Chiang MC, Knowlton BJ, Iacoboni M, Udompholkul P, et al. (2013) Interleaved practice enhances skill learning and the functional connectivity of fronto-parietal networks. Hum Brain Mapp 34: 1542-1558.

30. Siebner HR, Rossmeier C, Mentschel C, Peinemann A, Conrad B (2000) Short-term motor improvement after sub-threshold $5-\mathrm{Hz}$ repetitive transcranial magnetic stimulation of the primary motor hand area in Parkinson's disease. J Neurol Sci 178: 91-94.

31. Khedr EM, Rothwell JC, Shawky OA, Ahmed MA, Hamdy A (2006) Effect of daily repetitive transcranial magnetic stimulation on motor performance in Parkinson's disease. Mov Disord 21: 2201-2205. 